\title{
Les interactions verbales dans les émissions télévisuelles algériennes
}

\begin{abstract}
:
Our researchwork examines the specific features of mediated verbal interactions in Algerian televisions. Itsprimary aim is to study code switching and discoursecooperation in four TV programs: ' Paroles de Femmes', 'A CœurOuvert ', 'Bonjour d'Algérie ' in Canal Algerie TV and 'Controverse' in Dzair TV. Afterrecording and transcribing phonetically eightseries of the cited TV programs, weadopted the interdisciplinary inductive approachto analyse our corpus. Thus, the results of ouranalysis revealed that participants alternateFrench, Algerian Arabicdialect, English and Tamazight in theirmediated interactions in order to co-construct their verbal discourse, intended to bebroadcasted to the general public.
\end{abstract}

Key words: Verbal interactions, language practices, code-switching, discourse collaboration, Algerian TV

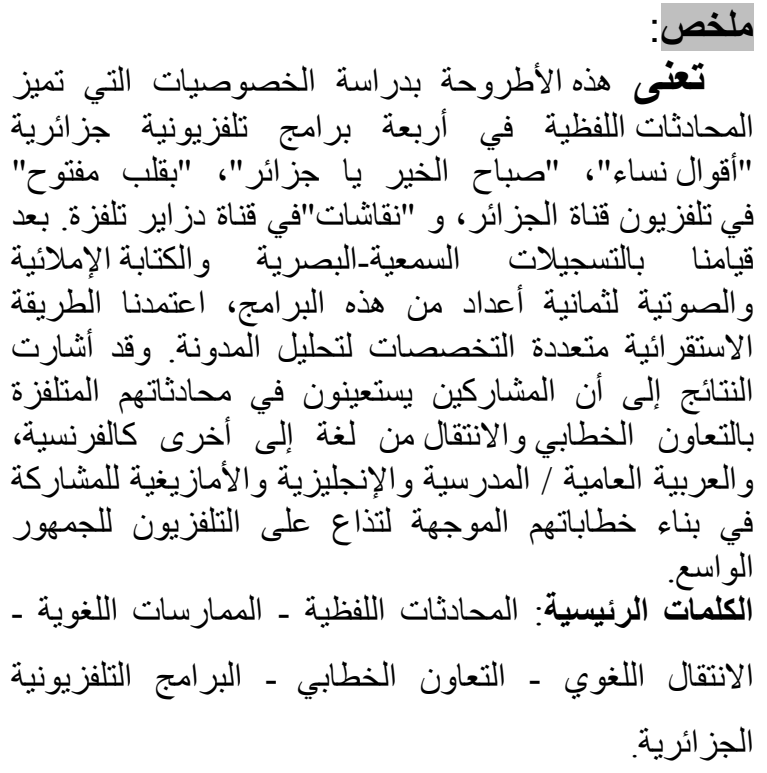

\author{
Aldjia OUTALEB- PELLÉ(1) \\ Amina Amel AZIL ${ }^{(2)}$ \\ Département des lettres et \\ langue française \\ Université Mouloud MAMMERI \\ de Tizi Ouzou ${ }^{(1)}$ \\ Université des Frères Mentouri \\ Constantine $^{(2)}$
}

\section{Introduction : \\ Les analogies « impressionnantes » entre les extensions technologiques, le langage et l'interaction sociale ont beaucoup contribué à l'étude du langage médiatique et des interactions verbales dans les programmes médiatisés. Ainsi, les études sur les interactions verbales et l'analyse de la conversation s'intéressent aujourd'hui à la question: «comment différentes audiences interprètent le message présenté à la radio et à la télévision? » Cet intérêt au discours diffusé à distance s'est développé surtout au}


Royaume-Uni, et s'est inspiré d'études littéraires et de la philosophie continentale européenne pour construire une approche dans laquelle les émissions diffusées sont considérées comme «des textes » que le public (l'audience) doit « lire ».

En Algérie, les mass-médias, institutions prépondérantes de l'Etat, ont connu ces dernières années une prolifération massive de chaînes télévisées. Qu'elles soient étatiques ou privées, ces chaînes diffusent des programmes en langues arabe, française, anglaise, et tamazight. Les travaux de recherche sur les phénomènes sociolinguistiques algériens dans les médias semblent se focaliser essentiellement sur l'étude de l'alternance codique, du fait que les interactants algériens sont plurilingues et que l'espace médiatique est, a fortiori, un lieu de production des pratiques langagières médiatisées.

Dans la présente étude, nous nous intéressons aux spécificités caractérisant la structure globale et locale des discours interactifs oraux se produisant dans un studio de télévision algérienne,des discours produits par des interlocuteurs en situation de face-à-face, mais destinés à être diffusés à un public. Nous allons examiner les productions interactionnelles des locuteurs algériens invités dans les quatre différentes émissions télévisées : «Bonjour d'Algérie », "Paroles de Femmes » et «A Cœur Ouvert» sur la chaîne satellitaire algérienne Canal Algérie, chaîne étatique francophone et l'émission «Controverse» dans la chaîne Dzair Tv, chaîne privée.

Le centre d'intérêt de notre travail est l'analyse de la coopération- un genre interactif et une forme de discours- dans les interventions des locuteurs invités pour discuter des différents thèmes socioculturels et politiques. La première raison pour laquelle nous nous intéressons à cette forme de discours est qu'elle est fondamentale pour le fonctionnement des émissions télévisuelles qui «se déroulent dans une situation à caractère contractuel qui voit chacun des acteurs donner des marques de déférence, de bonne volonté et d'entraide(...)» (Vion, 1992, p.125). Pour Vion (1992), la coopération représente également la plate forme de ce genre de médias audio-visuels :

«Le débat, représente aujourd'hui avec sa variante du faceà-face télévisé, la version moderne du tournoi chevaleresque où tous les coups ne sont pas permis en raison d'un code de courtoisie. L'existence de règles constitue l'une des preuves de la coopérativité régentant ces joutes compétitives. »(p.126).

Ce qui nous intéresse ici, est de montrer que la coopération discursive joue un rôle moteur en faveur du fonctionnement interactif des dites émissions.

\section{Problématique et hypothèse}


Nous avons constaté que les émissions de télévision sont un lieu culturel et social et une situation de communication propice aux échanges interactifs où les ressources linguistiques et sociales des participants se mêlent toutes afin de rallier le bon déroulement d'une interaction télévisuelle. Sur la base de ce constat, nous posons une série de questions sur les langues et les stratégies discursives utilisées par les locuteurs de télévision algérienne pendant leur échange verbal:

- Quels sont les différents phénomènes langagiers qui interviennent dans la construction coopérative du discours des locuteurs des émissions algériennes?

- Quelles sont les règles qui sous-tendent le bon fonctionnement de leurs interactions verbales?

- Quelle (s) langue (s) utilisent-ils pour établir le contact lors de leurs différentes discussions destinées à être diffusées à un public ?

- S'ils disposent de plusieurs langues, quel est le rôle de chacune d'elles? Notre hypothèse est que les locuteurs des émissions télévisuelles algériennes pourraient utiliser, stratégiquement, plus d'une langue afin de coconstruire conjointement un discours coopératif.

\section{Objectifs de la recherche}

Nous avons analysé les productions interactionnelles des invités des émissions télévisées algériennes afin de nous enquérir sur les différents éléments linguistiques et sociaux participant à la coopérativité de leur discours. Aussi, le but de notre étude est-il de dévoiler l'outil linguistique avec lequel les locuteurs arrivent à transmettre l'information et à organiser leurs différentes conversations du face-à-face en situation de communication médiatisée. Cette étude se propose donc de réaliser :

- Une analyse socio-interactionnelle des différents phénomènes langagiers participants à la construction coopérative du discours dans des émissions de télévisions algériennes.

- Une analyse sociolinguistique des fonctions communicatives des langues utilisées en interactions médiatisées.

\section{Démarche méthodologique}

Notre démarche méthodologique a consisté en des enregistrements audiovisuels d'une série d'émissions télévisées algériennes: "Bonjour d'Algérie », « Paroles de Femmes », «A cœur ouvert » et « Controverse », que nous avons transcrites orthographiquement et phonétiquement, et cela en adoptant les conventions de transcription élaborées par Traverso, V. (1999). Le corpus de notre travail de recherche comprend les interactions de huit émissions enregistrées d'une durée de quinze (15) heures. Pour analyser ces émissions, nous avons opté pour une perspective d'analyse inductive- 
multidimensionnelleappropriée à la nature complexe et spécifique du langage médiatique.

Les enregistrements authentiques de l'émission "Controverse » nous ont été tirés des archives audiovisuelles de l'établissement de télévision Dzair Tv grâce à l'indulgence d'une journaliste ayant gracieusement accepté d'effectuer ce travail bien nécessaire pour notre étude.

\section{Résultats préliminaires obtenus}

\section{Les formes et les types d'alternance codique}

Le corpus collecté a révélé que les interactants de ces émissions alternent continuellement et d'une manière stratégique cinq langues : le français, l'arabe dialectal, l'arabe scolaire, le tamazight et l'anglais. Pour l'animateur, aussi bien que pour les invités, l'alternance sert à mieux établir le contact direct et à mieux transmettre l'information aux téléspectateurs. Les tenants du discours l'utilisent pour structurer le déroulement de l'interaction et s'assurer de la coordination de leurs différentes actions discursives disposées à être télédiffusées, et également pour s'adresser la parole et apporter plus de précisions sur les différents sous-sujets débattus dans l'émission. Ainsi, ne s'agit-il pas pour eux de faire de l'alternance codique une stratégie discursive servant à la construction d'un discours médiatique cohérent, fondé sur la coopération interactive.

Il est important de noter que la fréquence des formes d'alternance codique présente une différence de taille. Les formes les plus fréquentes sont l'alternance intra-phrastique, l'alternance de type prédicat-complément: (prédicat en arabe/ complément en français, prédicat en français/ complément en arabe), l'alternance de type nom-complément, l'alternance de type sujetprédicat. Nous supposons que cela est dû à la nature des fragments alternés qui s'interposent régulièrement dans la contexture interne des divers systèmes grammaticaux en présence: indicateurs de personnes, éléments déictiques, embrayeurs, adjectifs, adverbes, verbes, conjonctions de coordination et conjonction de subordination en arabe dialectal algérien ou mots techniques appartenant au domaine médiatique télévisuel en français et parfois en anglais : «la caméra», «le cadreur», "camerawoman». En effet, les occurrences constantes de ces formes d'alternance codique attestent que ces interlocuteurs possèdent une compétence plurilingue quasi-équilibrée. Ainsi, l'alternance estelle actualisée « librement entre deux éléments quelconques d'une phrase, (...) ordonnés de la même façon selon les règles de leurs grammaires respectives » (Poplack, 1988, p.32). En fait, nous pouvons dire que :

«Les échanges bilingues que nous avons examinés montrent en outre que l'alternance codique n'indique pas nécessairement une connaissance imparfaite des systèmes 
grammaticaux concernés. Relativement rares sont les passages où le changement de code est motivé par l'incapacité des locuteurs à trouver les mots pour exprimer ce qu'ils veulent dire dans l'un ou l'autre code. Dans bien des cas, l'information que fournit l'alternance pourrait tout aussi bien s'exprimer dans l'autre langue » (Gumperz, 1989, p.63).

Les formes d'alternance codique les moins présentes dans notre corpus sont l'alternance inter-phrastique et l'alternance de type proposition principale/ proposition subordonnée. S'agissant de l'alternance codique extra-phrastique, elle n'est notée que deux fois, au début de l'émission "A Cœur Ouvert», par l'usage de deux formes liturgiques «[bismileharaЂmeneraЂim]» $=$ «Au nomde Dieu le Miséricordieux, le très Miséricordieux », « [asalemuSalajkumwarahmatouallahitạalawabarakatu]»= «que la paix et la miséricordede Dieusoient sur vous»; et dans l'émission «Paroles de Femmes », par l'usage de l'expression idiomatique [mən babək :: mənəsbərąlik] $=$ « je ne t'aime pas, mais je ne peux pas me passer de toi ».

\section{Fonctions spécifiques de l'alternance des langues dans les émissions télévisuelles analysées}

En nous focalisant non seulement sur les éléments linguistiques mais aussi sur les aspects pragmatiques ressortant des émissions de notre corpus, nous avons ainsi pu faire apparaître les fonctions spécifiques suivantes de l'alternance des langues :

- La personnalisation du message, qui exhibe l'implication des interactants dans leur discours ; elle est présente quasiment dans toutes les pratiques linguistiques des locuteurs par l'utilisation des indicateurs de possession : [bənəsba lia] = « pour moi », [dyalna] = «notre », ou par des indicateurs de personnes comme [ hna $]=$ « nous » $[$ ana $]=\langle$ moi $\rangle$

- La réitération : elle est fréquemment utilisée par les locuteurs pour faire ressortir le message, clarifier le discours, se prémunir de la bonne appréhension des énoncés, et soutenir l'intercompréhension des différentes idéologies [jaSni] $=\langle$ c'est-à-dire $»,[b \varepsilon]]=\langle$ pour $»$;

- La citation: les locuteurs l'utilisent pour rapporter les paroles d'autrui ; elle est généralement exprimée par des verbes introducteurs en arabe dialectal [qalət] $=$ «elle a dit », [qatli] = «elle m'a dit», et accomplie en français ;

- La désignation d'un interlocuteur présent : elle sert aux interactants à adresser la parole à un parmi plusieurs interlocuteurs pouvant être présents dans l'interaction; elle se réalise par l'usage des termes 
d'adresse (un terme de respect ou le prénom de l'interlocuteur précédé ou non de la particule vocative [a].

- La désignation d'un interlocuteur absent : elle sert aux locuteurs à désigner une personne non-présente dans l'interaction, par l'usage des pronoms personnels $[$ huma $]=\langle$ ils $»,[$ hia $]=\langle$ elle $»$;

- La modalisation et la reformulation d'un message : pour spécifier ou distinguer l'essentiel de leurs constructions énonciatives, les locuteurs utilisent les indicateurs de reformulation [jạni] = "c'est-à-dire ", $[$ habit nqol] $=$ « je veux dire $»$;

- Le récit ou la narration : elle se manifeste lorsque les interlocuteurs dénotent ce qu'ils ont fait dans un reportage, un micro-trottoir, un feuilleton, ou une enquête ;

- La jointure du discours : elle est effectuée par l'usage des éléments syntaxiques servant à articuler les différentes parties du discours $[$ Tənit $]=\langle$ aussi $»,[$ ou $]=\langle$ et $»,[$ wəla $]=\langle$ ou $»,[\mathrm{~b} \varepsilon f]=\langle$ pour $»$;

- Le mixage de codes : il consiste à transférer des segments verbaux de la langue arabe vers la langue française. Il s'effectue à travers les verbes par l'addition des affixes de l'arabe agglutinés à la racine des verbes français, [madəpsawəઈ] = "elles n'ont pas dépassé », [matakseptef] = «elle n'accepte pas », dans les noms par l'ajout du déterminant arabe $« \mathrm{el} », \ll 1 »$ aux termes français, [l'séparation] = « le choix », [el vide] = « le vide »;

- La coopération discursive : elle permet aux locuteurs de marquer leur connivence par l'usage des traits d'approbation [əh] = «oui », [sah] $=$ «c'est vrai»; et ainsi de contribuer promptement à la coénonciation du discours.

\section{Phénomènes de coopération discursive dans les émissions télévisées}

Les locuteurs engagés dans les interactions télévisuelles produisent des structures langagières ajustées afin de co-construire conjointement leur discours. L'analyse de ces structures, qui se base essentiellement sur les concepts théoriques et méthodologiques présentés par V.André (2006), R.Vion (1992) et C. Kerbrat-Orecchioni (1990), nous a permis d'identifier l'ensemble des phénomènes coopératifs présents dans notre corpus:

- l'énonciation conjointe : elle se manifeste comme une composante interactive formée de la succession de deux unités dialogiques. La seconde unité se situe constamment dans l'allongement interactif ou syntagmatique de la première ;

- complétion hétéro-déclenchée: pour poursuivre son discours,le premier interactant invite ses partenairesà l'aider à construire son tour de parole par complétion. Cette sollicitation peut être déclenchée explicitement par des marques interrogatives «comment dire?» ou 
implicitement par des hésitations, des bribes, des amorces, ou des répétitions de segments grammaticaux de liaison « de », «que »;

- complétion auto-déclenchée : elle est utilisée par les interlocuteurs pour faire avancer le discours et mener à bien l'enchaînement des différentes rubriques de l'émission; elle se manifeste comme une prétention de complétion actualiséepar le second locuteur, alors que le premier n'a fourni aucun signe sollicitant la co-production de son énoncé.

- ratification de la complétion par reprise : elle se présente sous forme d'un échange ternaire entre deux locuteurs. Le premier locuteur prononce un discours achevé volontairement par le second locuteur. Ensuite, le premier locuteur participe, par réitération, à cette action discursive pour admettre, approuver et confirmer la complétion de son co-énonciateur.

- reprise par répétition: elle est constatée lorsque les paroles complétives du second locuteur sont reprises par le premier locuteur sans aucune transformation lexicale;

- reprise par reformulation : elle sert au premier locuteur à ratifier la complétion du second en paraphrasant le contenu sémantique de la séquence discursive antérieure ;

- auto-reprise réitérative: elle est effectuée par le même locuteur reprenant ses propos pour réparer l'éventuelle incompréhension de la première énonciation occasionnée par le chevauchement de paroles;

- reprise diaphonique réitérative: elle est produite par le premier locuteur pour valider la contribution coopérative du second. Cette validation est totale, partielle ou modifiée ;

- marqueurs de convergence : pour soutenir l'énoncé formulé par un interlocuteur et, par la même occasion, souligner leur accord avec ce dernier, les locuteurs utilisent les termes d'acquiescement «c'est vrai », « exactement », « oui », « voilà », « bien sûr », [əh] = « oui », $[s a \hbar]=\langle$ c'est vrai $» ;$

- procédés phatiques: ils se manifestent par l'usage des captateurs « hein! », «n'est ce pas? », « vous voyez? », par l'énonciateur pour garantir l'écoute de son interlocuteur.

- éléments régulateurs: ils sont remarqués, en général, lorsque le récepteur produit des signaux d'écoute (hochements de tête, regards, vocalisation « $\mathrm{mmh} »)$ pour répondre à une sollicitation de l'énonciateur (élément phatique).

Visés et intentions communicatives des différents participants aux émissions 
Pour mieux cerner les intentions communicatives des différents participants aux émissions, nous nous sommes fondée sur les éléments pragmatiques liés aux fonctions socioculturelles et interactives des langues utilisées. Ceci nous a permis de mettre à jour quatre fonctions principales :

- les participants se servent des langues afin d'organiser le fil des différentes rubriques de l'émission. Cette organisation repose généralement sur un certain nombre de règles précises et de contraintes qui suggestionnent et orientent les actions interactionnelles des interlocuteurs; et donc qui assurent la maintenance de l'interaction: le prochain locuteur commence à parler lorsque le précédent s'arrête, les invités approuvent le rôle conversationnel de l'animateur qui occupe la fonction du gestionnaire officiel de l'échange interactif de l'émission, égard au relais des tours de parole naissant d'un consensus négocié au cours de l'interaction ;

- ils visent à créer une atmosphère de convivialité leur permettant l'élaboration et la construction coopérative du discours ;

- ils marquent leur appartenance à la religion musulmane et à la culture arabo-algérienne en utilisant des expressions liturgiques et des termes culturels en arabe dialectal ou scolaire ;

- ils s'appuient, pour préserver la face d'un interlocuteur, sur des stratégies de réparation ayant en vue de montrer aux téléspectateurs que ce dernier est susceptible de construire seul son discours.

\section{Structure globale de l'interaction médiatique}

S'agissant de l'organisation globale des émissions télévisées que nous avons étudiées, elle se décompose en sept parties : la présentation de l'émission, la pré-ouverture, l'ouverture, la réouverture, le corps, la pré-clôture, et la clôture. -La présentation de l'émission: elle sert, pour l'animateur, à présenter aux téléspectateurs le cadre général de l'émission et l'identité des invités.

-La pré-ouverture : elle correspond à l'accueil des participants et à l'échange des premières salutations entre l'animateur et les invités (termes de salutations, de politesse, questions sur l'état de santé, expression de bienvenue, etc.).

-L'ouverture : elle se rapporte à la prise de contact entre les interactants, « mais aussi et surtout à l'établissement d'une première définition » (Traverso, 1999, p.32)du thème à discuter au cours de l'interaction.

-La réouverture: elle correspond à la mise en relation des membres de l'émission qui s'orientent dans cette phase vers deux identités distinctes, celle d'animateur, et celle de participant. Ces derniers forment ainsi une paire relationnelle dans laquelle l'animateur informe les participants sur l'organisation de l'échange télévisé et surtout fait un rappel des règles du jeu interactif. 
-Le corps de l'interaction: il comprend un nombre indéfini et variable de séquences interactives qui occupent la totalité du mouvement discursif. Il représente le déroulement de l'interaction qui débute lorsque la séquence de réouverture s'achève.

-La pré-clôture : elle correspond à la pré-fermeture de l'interaction et donc à l'avant-fin de l'émission. Cette première phase de clôture de l'émission est indiquée par l'animateur par des expressions comme «nous arrivons donc à la fin de l'émission », «alors justement pour cette fin de partie de l'émission nous allons avoir un tête-à-tête ... mais avant ça je vous propose de suivre un micro-trottoir », « nous allons clore ce débat », pour préparer ses invités ainsi que ses téléspectateurs à la fin de l'émission.

-La clôture : elle comprend des actes spécifiques de clôture qui correspondent à la fermeture immédiate de l'interaction. Ces actes sont des salutations, des remerciements ou des prises de rendez-vous pour un nouvel épisode.

A l'issue de notre étude, nous pouvons dire que les interactions verbales dans les émissions télévisuelles algériennes étudiées se caractérisent, essentiellement, par la coopération discursive permettant aux participants de co-construire conjointement leur discours médiatique. Les mouvements coopératifs réalisés par la complétion, l'énonciation conjointe, la reprise, les procédés phatiques et les régulateurs facilitent la co-élaboration du discours dans une interaction polylogale regroupant plus de quatre interactants. Ainsi, cette analyse montre que l'alternance codique domine les pratiques langagières des participants aux émissions télévisuelles algériennes et que le français est la langue véhiculaire, voire la langue de la communication télévisée. L'arabe sert à articuler le discours et à marquer l'appartenance des membres à la culture arabo-musulmane.

\section{Bibliographie}

ANDRE, V. (2006).Construction collaborative du discours au sein de réunions de travail

en entreprise, de l'analyse micro-linguistique à l'analyse sociointeractionnelle, thèse

de doctorat, Nancy 2.

GUMPERZ, J-J. (1989a). Sociolinguistique interactionnelle. Une approche interprétative,

Paris : L'Harmattan.

GUMPERZ, J-J. (1989b). Engager la conversation, Paris : Minuit.

GUMPERZ, J-J.(2001).Interactional Sociolinguistics:A Personal Perspective, Oxford, Basil

Blackwell. 
KERBRAT-ORECCHIONI, C. (1998). « La notion d'interaction en linguistique : origine, apports, bilan », Langue française, vol. 117, $\mathrm{n}^{\circ} 1$, pp. 51-67.

POPLACK, S. (1988 a). "Conséquences linguistiques du contact de langues : un modèle d'analyse variationniste », in, Langage et société, $\mathrm{n}^{\circ} 43$, pp. 23 46.

POPLACK, S. (1988 b). «The social correlates and linguistic processes of lexical borrowing and assimilation » in, Linguistics, ${ }^{\circ} 26$, pp. 47-104.

QUFFELEC, A. DERRADJI, Y. DEBOV, V. SMAALI- DEKDOUK, D. CHERRAD-

\&BENCHEFRA. Y. (2002), Le français en Algérie. Lexique et dynamique des langues, Bruxelles : Duculot.

TRAVERSO, V. (1999). L'analyse des conversations, Paris : Nathan.

VION, R. (1992). La communication verbale:analyse des interactions, Paris : Hachette.

ZONGO, B. (1996). «Alternance des langues et stratégies langagières en milieu

d'hétérogénéité culturelle : vers un modèle d'analyse » in, Caroline JUILLARD \& Louis-Jean CALVET (dirs.), Les politiques linguistiques, mythes et réalité, Beyrouth, AUPELF-UREF, pp. 341349. 